\title{
Variables de la desnutrición en pacientes en diálisis
}

\author{
Luis Garrido Pérez, María Sanz Turrado, Carmen Caro Domínguez \\ Facultad de Medicina y Enfermería de Córdoba. Universidad de Córdoba. España
}

\section{Resumen}

Introducción: El paciente en diálisis va a sufrir una desnutrición proteico-calórica, con diferentes factores implicados en su aparición, lo cual se asocia con una elevadísima morbilidad cardiovascular y mortalidad. Se ha estimado una prevalencia de desnutrición en la población en hemodiálisis del $18-75 \%$, siendo por tanto, un problema de especial relevancia en este tipo de pacientes.

Objetivo: Realizar una revisión bibliográfica de los artículos científicos existentes sobre las variables que intervienen en la desnutrición del paciente en diálisis.

Metodología: Se ha realizado una revisión bibliográfica mediante las bases de datos PubMed, Scielo, ProQuest. La búsqueda se ha realizado con términos Mesh, con una antigüedad no mayor de 5 años y con distintas palabras clave.

Resultados: Se han revisado 19 artículos. La mayoría de los artículos fueron estudios observacionales y de revisión. Los factores que se asocian con desnutrición son la edad, pérdida de masa muscular, baja actividad física y dieta pobre en micronutrientes. Otro factor muy importante, es la inflamación. En cuanto a los métodos diagnósticos, son variados y diferentes, debido a la gran cantidad de variables que influyen en la desnutrición.

Conclusiones: La desnutrición en pacientes en diálisis depende de distintas variables y no solamente de la dieta. Los factores que se asocian con desnutrición son mayor edad, pérdida de masa muscular, baja actividad física y dieta pobre en micronutrientes. Además, habría que añadir el doble papel que juega la inflamación en

\footnotetext{
Correspondencia:

Luis Garrido Pérez

Facultad de Medicina y Enfermería. Universidad de Córdoba Avda. Menéndez Pidal s/n. 14004 Córdoba. España

E-mail:n22gapel@uco.es
}

este proceso, pues puede ser tanto consecuencia como factor predisponente a la desnutrición.

PALABRAS CLAVE

- DESNUTRICIÓN

- DIÁLISIS

- HEMODIÁLISIS

- DIÁLISIS PERITONEAL

- INFLAMACIÓN

- DESGASTE PROTEICO-ENERGÉTICO

\section{Variables of malnutrition in dialysis patients}

\section{Abstract}

Introduction: The patient on dialysis will suffer from protein-caloric malnutrition, with different factors involved in its onset, which is associated with very high cardiovascular morbidity and mortality. A prevalence of malnutrition in the hemodialysis population of 18$75 \%$ has been estimated, being therefore a problem of special relevance in this type of patients.

Objective: A literature review of the existing scientific articles on the variables involved in malnutrition of patients on dialysis was carried out.

Methods: A bibliographic review has been done using the PubMed, Scielo, ProQuest databases. The search used Mesh terms, with an age of no more than 5 years and with different keywords.

Results: Nineteen articles were reviewed. Most articles were observational and review studies. The factors that are associated with malnutrition are age, loss of muscle mass, low physical activity and diet deficient in micronutrients. Another very important 
factor is inflammation. Regarding the diagnostic methods are varied and different, due to the large number of variables that influence malnutrition.

Conclusions: Malnutrition in dialysis patients depends on different variables and not only on the diet. The factors that are associated with malnutrition are older age, loss of muscle mass, low physical activity and diet deficient in micronutrients. In addition, we should add the dual role of inflammation in this process as it can be both a consequence and a predisposing factor to malnutrition.

\section{KEYWORDS}

- MALNUTRITION

- DIALYSIS

- HEMODIALYSIS

- PERITONEAL DIALYSIS

- INFLAMMATION

- PROTEIN-ENERGY DEPLETION

\section{Introducción}

La enfermedad renal crónica avanzada (ERCA) se caracteriza por alteraciones nutricionales e inflamación sistémica que se acompaña de un aumento del catabolismo, lo que incrementa la morbimortalidad ${ }^{1}$. La desnutrición calórico-proteica es un problema muy frecuente entre los pacientes en hemodiálisis (HD), siendo, junto a la inflamación, los factores no tradicionales más potentes de riesgo cardiovascular de estos pacientes por el desarrollo de aterosclerosis ${ }^{2,3}$. Estos tres síntomas han sido referidos dentro del síndrome MIA (malnutrición-inflamación-aterosclerosis), el cual se asocia con una elevadísima morbilidad cardiovascular y mortalidad en los pacientes en $\mathrm{HD}^{4,5}$. Por otra parte, se ha estimado una prevalencia de desnutrición en la población en HD del 18-75 \% ${ }^{6}$, siendo por tanto, un problema de especial relevancia en este tipo de pacientes.

La malnutrición calórico-proteica se produce por el propio fracaso de la función renal, al producirse un aumento de factores neuroendocrinos y de citoquinas. Esta alteración hormonal produce hipertrigliceridemia y una alteración del metabolismo hidrocarbonado, con resistencia a la insulina que puede finalizar en un cuadro de diabetes ${ }^{7}$. El aumento de productos nitrogenados y las alteraciones iónicas producen trastornos gastrointestinales que reducen la ingesta, con náuseas y vómi- tos ${ }^{8}$. La diálisis también puede condicionar la situación nutricional, no debemos olvidar que los pacientes tratados con HD tienen un consumo proteico mayor que en la diálisis peritoneal, además existe mayor riesgo de déficit de vitaminas hidrosolubles y de hierro.

Por tanto, no existe una única definición de la desnutrición del paciente en HD, porque como hemos visto son varios los factores implicados en su aparición; y por ello se expresa como la consecuencia de una desproporción entre el gasto y la ingesta de proteínas y de calorías de largo periodo, en pacientes dializados ${ }^{9}$, es decir, como lo define Gracia-Iguacel, C. como 'un estado patológico donde hay un descenso o desgaste continuado tanto de los depósitos proteicos como de las reservas energéticas $^{\prime 10}$.

A pesar de que en estas definiciones se incide solamente en el gasto y la ingesta de proteínas y calorías, la desnutrición no es identificada solo por uno o dos marcadores, por ello hay que tener una visión más amplia para poder identificar los distintos factores asociados a este problema ${ }^{11}$. Los principales factores a tener en cuenta son el ejercicio físico, la anorexia y, por supuesto, la dieta, además de marcadores de riesgo como son los niveles de albúmina sérica, los niveles de proteína C-reactiva, el género y la edad ${ }^{12,13}$.

Las principales consecuencias de la desnutrición en los pacientes en HD van a ser por tanto, el aumento de la morbilidad y de la mortalidad ${ }^{14,15}$. Además, van a presentar alteraciones en los parámetros nutricionales y en sus medidas antropométricas con pérdida de peso corporal; y con marcadores inflamatorios altos entre el $30-65 \%$ de los pacientes con diálisis mantenida ${ }^{16}$.

Por todo ello, nos parece relevante el estudio de la malnutrición del paciente en diálisis, puesto que es una alteración compleja, multifactorial y que desemboca en gran medida, en otras complicaciones, empeorando el pronóstico y aumentando la morbimortalidad de estos pacientes.

Por consiguiente y con el objetivo general de realizar una revisión bibliográfica de los artículos científicos existentes sobre las variables que intervienen en la desnutrición de los pacientes en diálisis, nos planteamos los siguientes objetivos específicos:

- Determinar los factores de riesgo asociados a la desnutrición de pacientes en diálisis.

- Analizar las consecuencias de la desnutrición de estos pacientes. 


\section{Metodología}

Se ha llevado a cabo un estudio de revisión bibliográfica, para lo que se ha realizado una búsqueda sistemática en las distintas bases de datos PubMed, Scielo, ProQuest, con la finalidad de recopilar la bibliografía científica más idónea existente sobre este campo, de los últimos 5 años (2011-2016). La búsqueda se realizó durante el período de 22 de enero de 2016 hasta el 9 de marzo de 2016.

La búsqueda se realizó con distintas palabras clave: desnutrición (malnutrition), diálisis (dialysis), hemodiálisis (renal dialysis), diálisis peritoneal (peritoneal dialysis), inflamación (inflammation), desgaste proteico energético (protein-energy waste). Estas palabras clave se asociaron entre sí gracias a conectores boleanos (and, or, not) con lo cual se encontraron artículos más específicos. Al recopilar todos los artículos importantes sobre este tema, se realizó una búsqueda del factor de impacto de las revistas de las que fueron extraídos, para así poder organizarlos según cuartiles (Q1, Q2, Q3 y Q4), tanto en Journal Citation Report (JCR) como en SCimago Journal Rank (SJR). Los artículos de revistas que no se encontraban en estos repositorios fueron nuevamente revisados para aceptarlos o no, en función de los criterios de inclusión. Se aceptaron artículos tanto en inglés como en castellano.

\section{Criterios de inclusión}

- Artículos originales y de revisión que estudiaran las causas de la desnutrición de los pacientes adultos en diálisis.

- Artículos que analizaran los factores de riesgo de la desnutrición en pacientes en diálisis.

- Artículos que trataran sobre el desgaste proteico-energético del paciente en diálisis.

- Artículos que relacionaran inflamación y desnutrición de los pacientes en diálisis.

\section{Resultados y discusión}

\section{Búsqueda y primera selección de documentos}

Los documentos preseleccionados en cada base de datos en las que fue realizada la búsqueda se encuentra reflejado en la Tabla 1.
Tabla 1. Publicaciones encontradas y preseleccionadas en cada base de datos.

\begin{tabular}{l|c|c|c|c}
\hline Bases de datos & ProQuest & Pubmed & Scielo & Total de publicaciones \\
\hline $\begin{array}{l}\text { Publicaciones } \\
\text { Encontradas }\end{array}$ & 418 & 194 & 36 & 648 \\
\hline $\begin{array}{l}\text { Publicaciones } \\
\text { preseleccionadas }\end{array}$ & 26 & 25 & 1 & 52 \\
\hline
\end{tabular}

De los documentos preseleccionados se eliminaron aquellos que se repetían en varias bases de datos, siendo el resultado final de 52 artículos.

\section{Análisis de los resultados}

Se realizó una lectura de los resúmenes de los 52 artículos preseleccionados. Al realizar esta lectura se descartaron aquellos que no cumplían los requisitos del estudio quedándose así una selección de 41 artículos. Tras esta preselección, se realizó una lectura de los artículos para poder limitar mejor la cantidad de artículos a analizar y tener una visión más específica y de mejor calidad del problema de estudio. Debido a ello, se realizó de nuevo otra preselección de 27 artículos.

Finalmente, tras una nueva revisión de los artículos y con un mayor conocimiento sobre la información que contenían, la selección final fue de 19 artículos (Tabla 2). 
Tabla 2. Características de los artículos seleccionados.

\begin{tabular}{|c|c|c|c|}
\hline Autor, año, país, cuartil. & Muestra & Diseño del estudio & Conclusiones principales \\
\hline $\begin{array}{l}\text { Fouque D, Pelletier S, Mafra } \\
\text { D, Chauveau P. 2011, Francia } \\
\text { JCR: Q1, SJR: Q1 }\end{array}$ & & Revisión & $\begin{array}{l}\text { La ingesta inadecuada de proteínas } \\
\text { y la masa muscular baja ayudan a } \\
\text { la desnutrición e inflamación de los } \\
\text { pacientes en diálisis }\end{array}$ \\
\hline $\begin{array}{l}\text { Hasheminejad N, Namdari } \\
\text { M, Mahmoodi MR, } \\
\text { Bahrampour A, Azmandian J. } \\
\text { 2016, Irán }{ }^{17} \\
\text { JCR: Q4, SJR: Q3 }\end{array}$ & $\mathrm{N}=83$ & Estudio transversal & $\begin{array}{l}\text { La fuerza de presión manual debe } \\
\text { ser estudiada para comprobar } \\
\text { si puede ser incorporada como } \\
\text { herramienta útil y fiable para } \\
\text { evaluar la masa muscular en relación } \\
\text { al estado nutricional del paciente }\end{array}$ \\
\hline $\begin{array}{l}\text { Gracia-Iguacel C, González- } \\
\text { Parra E, Pérez-Gómez MV, } \\
\text { Mahíllo I, Egido J, Ortiz A. } \\
\text { 2013, España }{ }^{18} \\
\text { JCR: Q3, SJR: Q3 }\end{array}$ & $\mathrm{N}=122$ & Estudio observacional & $\begin{array}{l}\text { Existe relación entre la pérdida } \\
\text { de masa muscular, debido al } \\
\text { catabolismo proteico, y la } \\
\text { mortalidad }\end{array}$ \\
\hline $\begin{array}{l}\text { As'habi A, Tabibi H, } \\
\text { Nozary-heshmati B, } \\
\text { Mahdavi-mazdeh M, } \\
\text { Hedayati M. 2014, Irán }{ }^{19} \\
\text { JCR: Q3, SJR: Q3 }\end{array}$ & $\mathrm{N}=291$ & $\begin{array}{l}\text { Observacional prospectivo } \\
\text { randomizado }\end{array}$ & $\begin{array}{l}\text { En este estudio los resultados } \\
\text { indican que MIS es casi similar } \\
\text { a SGA para identificar desgaste } \\
\text { proteico energético en pacientes } \\
\text { hemodializados }\end{array}$ \\
\hline $\begin{array}{l}\text { Chen J, Peng H, Xiao L, } \\
\text { Zhang K, Yuan Z, Chen J, } \\
\text { 2013. China }{ }^{20} \\
\text { JCR: Q1, SJR: Q1 }\end{array}$ & $\mathrm{N}=75$ & $\begin{array}{l}\text { Estudio observacional } \\
\text { analítico }\end{array}$ & $\begin{array}{l}\text { La inflamación sería la principal } \\
\text { causa de desnutrición en pacientes } \\
\text { en HD y no la insuficiente ingesta } \\
\text { de macronutrientes }\end{array}$ \\
\hline $\begin{array}{l}\text { Koor BE, Nakhaie MR, } \\
\text { Babaie S. 2015. Irán²1 } \\
\text { JCR:- , SJR:Q2 }\end{array}$ & $\mathrm{N}=190$ & $\begin{array}{l}\text { Estudio descriptivo } \\
\text { transversal }\end{array}$ & $\begin{array}{l}\text { Según el SGA más de la mitad de } \\
\text { los pacientes en HD tiene una leve } \\
\text { desnutrición, estos datos indican } \\
\text { que una medición apropiada } \\
\text { del estado nutricional en estos } \\
\text { pacientes es importante }\end{array}$ \\
\hline $\begin{array}{l}\text { Martín-del-Campo F, Batis- } \\
\text { Ruvalcaba C, González- } \\
\text { Espinoza L, et al. 2012, } \\
\text { Méjico } \\
\text { JCR: Q3, SJR: Q2 }\end{array}$ & $\mathrm{N}=73$ & Estudio transversal & $\begin{array}{l}\text { Sobre la mitad de los pacientes } \\
\text { en diálisis peritoneal tienen una } \\
\text { ingesta inadecuada de hierro, zinc, } \\
\text { calcio, vitaminas } A, B 6, C, \text { niacina y } \\
\text { ácido fólico. } \\
\text { La baja ingesta de micronutrientes } \\
\text { es relacionada con inflamación y } \\
\text { desnutrición }\end{array}$ \\
\hline $\begin{array}{l}\text { Carrero JJ, Stenvinkel } \\
\text { P, Cuppari L, Ikizler TA, } \\
\text { Kalantar-Zadeh K, Kaysen } \\
\text { G, 2013. Gran Bretaña }{ }^{23} \\
\text { JCR: Q3, SJR: Q2 }\end{array}$ & & Revisión & $\begin{array}{l}\text { La desnutrición es causada por } \\
\text { multitud de variables como la } \\
\text { dieta, las comorbilidades, las } \\
\text { medidas antropométricas y } \\
\text { composición corporal, entre otras }\end{array}$ \\
\hline $\begin{array}{l}\text { Ikizler TA, Cano NJ, Franch } \\
\text { H, Fouque D, Himmelfarb } \\
\text { J, Kalantar-zadeh K. 2013, } \\
\text { EE.UU. } \\
\text { JCR: Q1, SJR: Q1 }\end{array}$ & & Revisión & $\begin{array}{l}\text { La pérdida de nutrientes durante } \\
\text { la diálisis afecta significativamente } \\
\text { a la desnutrición, por ello hay que } \\
\text { estudiar las sesiones de diálisis que } \\
\text { hay que prescribir }\end{array}$ \\
\hline
\end{tabular}




\begin{tabular}{|c|c|c|c|}
\hline Autor, año, país, cuartil. & Muestra & Diseño del estudio & Conclusiones principales \\
\hline $\begin{array}{l}\text { Rattanasompattikul M, } \\
\text { Molnar MZ, Zaritsky JJ, et } \\
\text { al, 2013. EE.UU. } \\
\text { JCR: Q1, SJR: Q1 }\end{array}$ & $\mathrm{N}=754$ & Estudio cohortes & $\begin{array}{l}\text { El complejo desnutrición- } \\
\text { inflamación es un predictor de } \\
\text { una pobre respuesta a agentes } \\
\text { estimulantes de la eritropoyetina } \\
\text { en pacientes en HD }\end{array}$ \\
\hline $\begin{array}{l}\text { Quero A, Fernández R, } \\
\text { Fernández R, Gómez FJ, } \\
\text { 2015. España } \\
\text { JCR: Q4, SJR: Q2 }\end{array}$ & $\mathrm{N}=90$ & Observacional prospectivo & $\begin{array}{l}\text { La albúmina sérica es un } \\
\text { marcador de gran importancia } \\
\text { para la predicción y detección } \\
\text { de desnutrición en pacientes en } \\
\text { diálisis }\end{array}$ \\
\hline $\begin{array}{l}\text { Demirci MS, Demirci C, } \\
\text { Ozdogan 0, Kircelli F, } \\
\text { Akcicek F, Basci A. 2011, } \\
\text { Turquía }{ }^{27} \\
\text { JCR: Q1, SJR: Q1 }\end{array}$ & $\mathrm{N}=95$ & Observacional descriptivo & $\begin{array}{l}\text { Hay relación entre el agua } \\
\text { extracelular/peso y una nutrición } \\
\text { pobre, inflamación, ateroesclerosis } \\
\text { y sobrecarga de fluidos en } \\
\text { pacientes con diálisis peritoneal }\end{array}$ \\
\hline $\begin{array}{l}\text { Kovesdy CP, Kalantar- } \\
\text { Zadeh K, 2012. EE.UU. }{ }^{28} \\
\text { JCR: Q3, SJR: Q2 }\end{array}$ & & Revisión & $\begin{array}{l}\text { La falta de un test formal bien } \\
\text { definido para el desgaste proteico } \\
\text { energético hace que el diagnóstico } \\
\text { exacto sea imposible. Usar una } \\
\text { combinación de criterios podría } \\
\text { medir diferentes aspectos de este } \\
\text { desgaste }\end{array}$ \\
\hline $\begin{array}{l}\text { Toledo FR, Antunes AA, } \\
\text { Vannini FC, D., Silveira A. } \\
\text { 2013, Brasil' } \\
\text { JCR: Q3, SJR: Q3 }\end{array}$ & $\mathrm{N}=106$ & Estudio prospectivo & $\begin{array}{l}\text { El sistema de puntuación propuesto } \\
\text { por International Society of } \\
\text { Renal Nutrition and Metabolism } \\
\text { identifica mejor a los pacientes con } \\
\text { alto riesgo de muerte }\end{array}$ \\
\hline $\begin{array}{l}\text { Anand S, Chertow GM, } \\
\text { Johansen KL, et al. 2011, } \\
\text { EE.UU. }{ }^{30} \\
\text { JCR: Q2, SJR: Q2 }\end{array}$ & $N=266$ & Observacional de cohortes & $\begin{array}{l}\text { Los pacientes en diálisis con bajos } \\
\text { niveles de actividad física son } \\
\text { propensos a sufrir desnutrición y/o } \\
\text { inflamación }\end{array}$ \\
\hline $\begin{array}{l}\text { Sezer S, Bal Z, Tutal E, } \\
\text { Uyar ME, Acar N. 2014, } \\
\text { Turquía }{ }^{31} \\
\text { JCR: Q2, SJR: Q1 }\end{array}$ & $N=62$ & Casos control & $\begin{array}{l}\text { La suplementación nutricional } \\
\text { oral mejora el estado nutricional e } \\
\text { inflamatorio de los pacientes con } \\
\text { ERCA en HD }\end{array}$ \\
\hline $\begin{array}{l}\text { Chen J, Peng H, Zhang K, } \\
\text { Xiao L, Yuan Z, Chen J. } \\
\text { 2013, China }{ }^{32} \\
\text { JCR: Q1, SJR: Q1 }\end{array}$ & $\mathrm{N}=75$ & $\begin{array}{l}\text { Estudio multicéntrico } \\
\text { transversal }\end{array}$ & $\begin{array}{l}\text { La reducción de la ingesta de Se, } \\
\mathrm{Cu} \text { I y } \mathrm{Mn} \text { pueden ser marcadores } \\
\text { alarmantes para el estado de } \\
\text { malnutrición e inflamación en } \\
\text { pacientes en HD }\end{array}$ \\
\hline $\begin{array}{l}\text { Beberashvili I, Azar A, } \\
\text { Sinuani I, et al. 2013, } \\
\text { Israel }^{33} \\
\text { JCR: Q1, SJR: Q1 }\end{array}$ & $\mathrm{N}=75$ & $\begin{array}{l}\text { Estudio observacional } \\
\text { prospectivo }\end{array}$ & $\begin{array}{l}\text { MIS tiene menos reproducibilidad } \\
\text { interobservador que GNRI, pero es } \\
\text { más comprensible }\end{array}$ \\
\hline $\begin{array}{l}\text { Streja E, Kovesdy CP, } \\
\text { Molnar MZ, Norris KC, } \\
\text { Greenland S, Nissenson } \\
\text { AR. 2011, EE.UU. }{ }^{34} \\
\text { JCR: Q1, SJR: Q1 }\end{array}$ & $N=124029$ & Estudio de cohortes & $\begin{array}{l}\text { Las ventajas de supervivencia } \\
\text { de los pacientes afroamericanos } \\
\text { e hispanos en HD pueden estar } \\
\text { relacionadas con las diferencias en } \\
\text { el estado nutricional e inflamatorio }\end{array}$ \\
\hline
\end{tabular}

JCR: Journal Citation Report. SJR: SCimago Journal Rank. Q: cuartiles. N: número de sujetos de estudio. SGA: Subjective Global Assessment.

MIS: Malnutrition-Inflammation Score. GNRI: Geriatric Nutritional Risk Index. 


\section{Descripción de los resultados}

A pesar de las consecuencias y la prevalencia de la desnutrición en pacientes en diálisis, sigue sin haber un único método para su diagnóstico. Se han utilizado varios métodos de medida para el diagnóstico del desgaste proteico energético en pacientes en HD, los más importantes son el SGA (Subjective Global Assessment) y el MIS (Malnutrition-Inflammation Score). EI SGA es una herramienta clínica validada para el screening del riesgo nutricional de pacientes en $H D$, pero el MIS ${ }^{33}$ ha sido validado como mejor indicador nutricional, además de que parece ser el método más fiable para graduar el desgaste proteico energético ${ }^{17}$. EI mayor problema de SGA es que depende de la observación subjetiva, por ello es menos fiable que el MIS. Los artículos revisados utilizan distintos métodos para el diagnóstico, detección y medida de la malnutrición en pacientes en diálisis, como son los parámetros antropométricos, parámetros de laboratorio o el índice de masa corporal.

\section{Prevalencia}

La bibliografía revisada muestra un amplio rango de desnutrición en los pacientes en diálisis, este oscila en distintos estudios entre el 20 y el $80 \%^{16,17,18,19}$.

Este rango tan amplio puede ser debido a distintas causas tales como, tamaño muestral y tipo de estudio realizado, variabilidad geográfica, distintos grupos muestrales con distintas características, distintos métodos de evaluación de la desnutrición, ya que no hay un método único, distinto criterio para indicar la desnutrición.

\section{Edad}

Según varios estudios no hay una relación significativa entre la edad de los pacientes y la prevalencia de malnutrición ${ }^{18,20,21}$; sin embargo, hay otros estudios que indican que el estado nutricional de los pacientes de mayor edad es peor que el de los jóvenes ${ }^{22}$, lo cual es apoyado por el estudio transversal que indica que la fuerza de presión manual es un indicador de desnutrición en el cual se muestra que las personas de mayor edad tienen peores resultados que los de menor edad, debido a menor masa muscular ${ }^{17}$.

\section{Género}

El género es un factor de riesgo controvertido en relación a la desnutrición en diálisis ${ }^{18}$, ya que según el estudio transversal de Koor BE no hay diferencia significativa en los resultados del SGA según la edad ${ }^{21}$, aunque algunos autores señalan que las mujeres en diálisis tienen mayor porcentaje de desnutrición que los hombres ${ }^{22}$. Igual que ocurre con la edad, cuando se utiliza la fuerza de presión manual como indicador de la desnutrición, las mujeres obtienen peores resultados en esta prueba ${ }^{17}$.

\section{Anorexia}

La anorexia es uno de los principales efectos adversos de la ERCA, la cual produce una reducción de la ingesta de proteínas y calorías lo que contribuye a peor calidad de vida ${ }^{16,23}$. La anorexia tiene una prevalencia del $35 \%$ al $50 \%$ en pacientes con ERCA en estadío final $^{23}$. La anorexia se ve favorecida por la retención de toxinas urémicas ${ }^{23,24}$, el proceso de diálisis, enfermedades recurrentes, enfermedad cardiovascular, acidosis ${ }^{24}$ y la inflamación 22,24 .

\section{Pérdida de masa muscular}

La pérdida de masa muscular es una característica predominante en pacientes con ERCA y está particularmente presente en pacientes en HD periódicas ${ }^{16}$. Esta pérdida se ha asociado con mortalidad en estos pacientes $^{18}$. Esto puede ser debido a diversas causas; una de las más importantes es la inflamación que incrementa el catabolismo proteico y produce la pérdida de músculo ${ }^{22,23}$. Además, es una complicación derivada de la ERCA lo cual limita la capacidad de realizar ejercicio, a lo que habría que añadir los efectos catabólicos de la HD y las pérdidas de aminoácidos y proteínas que se producen durante las sesiones, provocando esto una baja disponibilidad de nutrientes para la síntesis muscular ${ }^{23}$. Finalmente, cabría añadir la comorbilidad de la diabetes, la cual agrava esta pérdida de masa muscular debido a la descomposición de proteínas causada por la falta o resistencia a la insulina ${ }^{23}$.

\section{Inflamación}

La bibliografía indica que la inflamación y la desnutrición suelen coexistir en pacientes en HD ${ }^{16,20,22,25,26}$, de los cuales el $53 \%$ de los que padecen malnutrición tienen signos de inflamación y el $72 \%$ de los pacientes con inflamación tienen signos de desnutrición ${ }^{20}$. En cualquier caso, la inflamación suele estar presente en la mayoría de los pacientes en HD. Un 30-65\% de los pacientes tienen un bajo grado de inflamación cróni$\mathrm{Ca}^{16}$, debido en parte al proceso de HD${ }^{25}$. 
En un estudio de pacientes en diálisis peritoneal se destaca la asociación entre desnutrición, inflamación y arterioesclerosi $\mathrm{s}^{27}$, lo cual se asocia con un aumento de riesgo de muerte cardiovascular ${ }^{28}$.

\section{Ejercicio físico}

La falta de ejercicio físico es un gran factor de riesgo para la desnutrición en pacientes en diálisis, tal como destacan diversos autores ${ }^{16,23,29,30}$. Los pacientes en HD tienen unos niveles muy bajos de actividad física ${ }^{30}$; además, hay que tener en cuenta que estos pacientes tienen menor actividad física los días que tienen sesión de diálisis, debido a la falta de movimiento que se produce durante la HD, la fatiga postdiálisis y el tiempo empleado en ir y volver de las sesiones ${ }^{16}$.

Algunos estudios destacan que un bajo nivel de actividad física del paciente en HD influye en la pérdida de masa muscular ${ }^{16,29}$, lo cual está asociado con un incremento de los marcadores inflamatorios ${ }^{23}$ y con un aumento de la mortalidad ${ }^{16,23}$.

Por todo ello, es de vital importancia fomentar la actividad física en estos pacientes, ya que está asociada con los marcadores nutricionales e inflamatorios ${ }^{30}$ presentando mejor pronóstico los pacientes que incrementan la actividad física.

\section{Dieta}

La dieta es un factor ampliamente analizado en los pacientes en diálisis, por su posible implicación en la desnutrición ${ }^{16,20,22,23,24,31,32}$. En la bibliografía se indica que la ingesta de calorías y proteínas en pacientes en diálisis es menor de la recomendada y que esto se asocia con un descenso de los parámetros nutricionales lo cual incrementa el riesgo de mortalidad y morbilidad $^{23,24}$, por ello algunos estudios confirman la mejora metabólica cuando los pacientes controlan su ingesta de proteínas y adaptan su ingesta calórica según sus requerimientos ${ }^{16}$.

Sin embargo, se ha señalado que la ingesta de proteínas y calorías no tiene asociación significativa con MIS $^{20,32}$ y en un estudio observacional se ha comprobado que no hay diferencias en la ingesta de calorías, proteínas, carbohidratos, fibra alimentaria y colesterol entre un grupo de pacientes de diálisis bien nutrido y otro desnutrido ${ }^{20}$. Este mismo estudio indica que donde se encuentra la diferencia es en la ingesta de micronutrientes, donde se produce una ingesta insuficiente ${ }^{20}$, esto es corroborado por otro estudio en el cual se in- dica que la ingesta de $\mathrm{Se}, \mathrm{Cu}$, I y $\mathrm{Mn}$ es inferior en un grupo de pacientes con desnutrición que en uno sin ella ${ }^{32}$. Además, esta menor ingesta de micronutrientes se asoció con malnutrición e inflamación ${ }^{32}$. Sin embargo, un estudio señala que tampoco los pacientes bien nutridos tienen la ingesta necesaria de micronutriente ${ }^{22}$, lo cual sugiere que un plan de cuidados nutricionales puede ser un factor protector, ya que se podría informar y ayudar a los pacientes para adaptar sus $\operatorname{dietas}^{16}$.

Un estudio de casos y controles ${ }^{31}$ muestra cómo la suplementación nutricional ha ayudado a mejorar significativamente los parámetros nutricionales en pacientes malnutridos en HD, además también han mejorado el estado inflamatorio, mientras que los pacientes que no tomaron esta suplementación empeoraron sus marcadores de desnutrición e inflamación en 6 meses $^{31}$.

\section{Morbilidad}

El desgaste proteico energético está relacionado con un incremento de la morbilidad en pacientes en diáli$\mathrm{sis}^{33}$. Se observa que el índice de resistencia a la eritropoyetina es un factor asociado a la desnutrición ${ }^{18}$ lo cual puede ser debido al descenso de la sensibilidad a los agentes estimulantes de la eritropoyesis causada normalmente por la anemia refractaria ${ }^{25}$. Cuando se analiza la desnutrición en pacientes con ERCA secundaria a diabetes, se ha observado que tienen una mayor incidencia de desgaste proteico energético cuando se les compara con sus homólogos sin diabetes ${ }^{24}$. Pero además, la diabetes es un predictor de la pérdida de masa muscular en pacientes en diálisis, ya que la falta de insulina o la resistencia a ella causa un incremento en la descomposición de proteínas musculares ${ }^{23}$. Además también contribuye a las neuropatías y a las enfermedades cardiovasculares que son una comorbilidad común ${ }^{23}$.

\section{Mortalidad}

La mortalidad es una consecuencia importante de la desnutrición en pacientes en diálisis, por ejemplo, en Estados Unidos alrededor de uno de cada 5 pacientes dializados muere cada año, y la supervivencia en 5 años es solamente de cerca del $35 \%{ }^{34}$, esto nos hace tener una idea de lo grave que es el problema de la desnutrición del paciente en diálisis y como, este factor incrementa la mortalidad en estos pacientes ${ }^{28,33}$. Según un estudio, los pacientes sedentarios que comienzan diálisis tienen un $60 \%$ más de mortalidad al año que sus homólogos activos físicamente ${ }^{30}$. 
Sin embargo, en otro estudio se señala que el desgaste proteico energético no es una causa directa de una mayor mortalidad, pero que contribuye a empeorar los efectos adversos de enfermedades cardiovasculares e infecciones, que son las causas más comunes de muerte en pacientes en $H^{29}$.

\section{Detección de la desnutrición}

La falta de un consenso para la medida del desgaste proteico energético es un problema para diagnosticarlo, ya que hay gran variedad de criterios diagnósticos potenciales que pueden ser empleados ${ }^{28}$. Por ello los sistemas de puntuación son problemáticos, porque cada uno tiene distintas variables y distintos resultados ${ }^{29}$.

Hay un estudio que sugiere que la fuerza de presión manual es un instrumento válido para el screening de la malnutrición e inflamación en pacientes en diálisis, ya que se ha asociado con MIS en un análisis monovariable, pero no es fiable cuando se indican más variables ni se asocia con $\mathrm{SGA}^{17}$.

Por ello las medidas más importantes según la bibliografía es el SGA y el MIS, ya que SGA es una herramienta clínica validada para el screening del riesgo nutricional de pacientes en HD y MIS ha sido validado como mejor indicador nutricional que SGA, pero MIS está basado en $S G A^{33}$. Pero habría que añadir que el SGA tiene un sistema semicuantitativo con solo tres niveles de severidad, mientras que MIS es totalmente cuantitativo ${ }^{19}$.

Además habría que nombrar que el plantel de expertos de la sociedad internacional del metabolismo y nutrición renal ha indicado que el diagnóstico del desgaste proteico energético podría hacerse con cuatro criterios $^{28}$ :

1.-Medidas bioquímicas (albúmina sérica, prealbúmina, transferrina y colesterol).

2.- Medidas de la masa corporal (IMC, pérdida de peso no intencionado y grasa total del cuerpo).

3.- Medidas de la masa muscular (masa muscular total, circunferencia del músculo de medio brazo y aparición de creatinina).

4.- Medidas de la ingesta dietética (ingesta dietética de proteínas y calorías).

Este grupo indicó que al menos 3 de las 4 categorías diagnósticas deben ser anormales para el diagnóstico del desgaste proteico energético.

\section{Consideraciones prácticas}

Como consideraciones prácticas señalar que, debido a la alta prevalencia de la malnutrición y sus consecuencias, del paciente en diálisis; todos los profesionales implicados en la asistencia sanitaria de estos pacientes, debe contribuir a la detección de la misma en estadíos precoces para prevenir su aparición. En este sentido, enfermería debe jugar un papel fundamental colaborando en el cuidado dietético/nutricional del paciente y realizando análisis del estado nutricional periódicamente con alguno de los instrumentos más utilizados como el SGA, que es sencillo de realizar y de los más fiables.

\section{Conclusiones}

A la vista de estos resultados, se puede concluir que la desnutrición en pacientes en diálisis depende de distintos factores y no solamente de la dieta como podría pensarse, siendo un problema bastante más complejo y de alta prevalencia.

Los factores que más influyen en la desnutrición en estos pacientes son mayor edad, la pérdida de masa muscular, baja actividad física, una dieta pobre en micronutrientes. En cuanto al género, no está claro su influencia en la desnutrición del paciente en diálisis.

Además, habría que añadir el doble papel que juega la inflamación en este proceso, pues puede ser tanto consecuencia como factor predisponente a la desnutrición.

A pesar de la existencia de distintos métodos diagnósticos no se utiliza un único método ya que debido a la gran cantidad de variables que influyen en la desnutrición, hay que utilizar diferentes pruebas diagnósticas, que en la mayoría de los casos son complementarias entre sí.

Por ello, una buena educación nutricional y en los estilos de vida de los pacientes junto con una detección precoz del problema podría prevenir o retrasar la desnutrición de los pacientes en diálisis.

Recibido: 28 octubre 2016

Revisado: 30 octubre 2016

Modificado: 4 noviembre 2016

Aceptado: 5 noviembre 2016 


\section{Bibliografía}

1. Fouque $D$, Vennegoor $M$, ter Wee $P$, Wanner $C$, Basci $A$, Canaud $B$, et al. EBPG guideline on nutrition. Nephrol Dial Transplant 2007;22 Suppl 2:ii45-87.

2. Stenvinkel $P$, Heimburger 0 , Paultre $F$, Diczfalusy $U$, Wang T, Berglund $L$, et al. Strong association between malnutrition, inflammation, and atherosclerosis in chronic renal failure. Kidney Int 1999;55(5):1899-911.

3. Kalantar-Zadeh K, Kopple JD, Humphreys MH, Block G. Comparing outcome predictability of markers of malnutrition-inflammation complex syndrome in haemodialysis patients. Nephrol Dial Transplant 2004;19:1507-19.

4. Dukkipati R, Kopple JD. Causes and prevention of protein-energy wasting in chronic kidney failure. Semin Nephrol 2009;29:39-49.

5. Chan M, Kelly J, Batterham M, Tapsell L. Malnutrition (subjective global assessment) scores and serum albumin levels, but not body mass index values, at initiation of dialysis are independent predictors of mortality: a 10-year clinical cohort study. J Ren Nutr 2012;22(6):547-57.

6. Leinig $C E$, Moraes $T$, Ribeiro $S$, Riella $M C$, Olandoski M, Martins $C$, et al. Predictive value of malnutrition markers for mortality in peritoneal dialysis patients. J Ren Nutr 2011;21:176-83.

7. Luis de D, Bustamante J. Aspectos nutricionales en la insuficiencia renal. Nefrología 2008;28(3):33948.

8. Pérez V0, Hernández EB, Bustillo GG, Penié $J B$, Porbén SS, Borrás AE et al. Nutritional status in chronic renal failure patients assisted at the hemodialysis program of the "Hermanos Ameijeiras» Hospital. Nutr Hosp 2007; 22:677-94.

9. Carrero JJ, Stenvinkel P, Cuppari L, Ikizler TA, Kalantar-Zadeh $K$, Kaysen $G$, et al. Etiology of the protein-energy wasting syndrome in chronic kidney disease: a consensus statement from the International Society of Renal Nutrition and Metabolism (ISRNM). J Ren Nutr 2013;23:77-90.

10. Gracia-Iguacel C, González-Parra E, BarrilCuadrado G, Sánchez R, Egido J, Ortiz-Arduán A, Carrero JJ. Definiendo el síndrome de desgaste proteico energético en la enfermedad renal crónica: prevalencia e implicaciones clínicas Nefrología 2014;34(4):507-19. doi:10.3265/Nefrologia. pre2014.Apr.12522.

11. Carrero JJ, Park SH, Axelsson J, Lindholm B, Stenvinkel P. Cytokines, atherogenesis, and hypercatabolism in chronic kidney disease: a dreadful triad. Semin Dial 2009;22:381-6.

12. Kalantar-Zadeh K, Block G, McAllister CJ, Humphreys MH, Kopple JD. Appetite and inflammation, nutrition, anemia, and clinical outcome in hemodialysis patients. Am J Clin Nutr 2004;80:299-307

13. Kalantar-Zadeh K, Ikizler TA, Block G, Avram $M M$, Kopple JD. Malnutrition-inflammation complex syndrome in dialysis patients: causes and consequences. Am J Kidney Dis 2003;42:864-81.

14. IkizlerTA, Wingar RL, Harvell J, ShyrY, Hakim RM. Association of morbidity with markers of nutrition and inflammation in chronic hemodialysis patients: A prospective study. Kidney Int 1999;55:1945-51.

15. Stenvinkel $P$, Heimbürger 0 , Lindholm B. Wasting, but not malnutrition, predicts cardiovascular mortality in end stage renal disease. Nephrol Dial Transplant 2004;19:2181-3.

16. Fouque D, Pelletier S, Mafra D, Chauveau P. Nutrition and chronic kidney disease. Kidney Int 2011 08;80(4):348-57. doi:10.1038/ki.2011.118

17. Hasheminejad N, Namdari M, Mahmoodi MR, Bahrampour A, Azmandian J. Association of Handgrip Strength With Malnutrition-Inflammation Score as an Assessment of Nutritional Status in Hemodialysis Patients. Iran J Kidney Dis. enero de 2016;10(1):30-5.

18. Gracia-Iguacel $C$, González-Parra $E$, Pérez-Gómez MV, Mahíllo I, Egido J, Ortiz A, et al. Prevalencia del síndrome de desgaste proteico-energético y su asociación con mortalidad en pacientes en hemodiálisis en un centro en España. Nefrología (Madrid). 2013;33(4):495-505. doi:10.3265/ Nefrologia.pre2013.Apr.11979

19. As'habi A, Tabibi H, Nozary-heshmati B, Mahdavimazdeh $M$, Hedayati $M$. Comparison of various scoring methods for the diagnosis of protein-energy wasting in hemodialysis patients. Int Urol Nephrol 
2014;46(5):999-1004. doi 10.1007/s11255-0130638-1.

20. Chen J, Peng H, Xiao L, Zhang K, Yuan Z, Chen J, et al. Inflammation but not dietary macronutrients insufficiency associated with the malnutritioninflammation score in hemodialysis population. PLoS ONE. 2013;8(12):e83233. doi:10.1371/ journal.pone.0083233.

21. Koor $B E$, Nakhaie $M R$, Babaie $S$. Nutritional assessment and its correlation with anthropometric measurements in hemodialysis patients. Saudi $\mathrm{J}$ Kidney Dis Transpl. agosto de 2015;26(4):697701.

22. Martín-del-Campo F, Batis-Ruvalcaba C, GonzálezEspinoza L, Rojas-Campos E, Ángel JR, Ruiz N, González J, Pazarín L, Cueto-Manzano AM. Dietary micronutrient intake in peritoneal dialysis patients:relationship with nutrition and inflammation status. Perit Dial Int. 2012 03;32(2): 183-91. doi: 10.3747/pdi.2010.00245.

23. Carrero JJ, Stenvinkel $P$, Cuppari L, Ikizler TA, Kalantar-Zadeh K, Kaysen $G$, et al. Etiology of the protein-energy wasting syndrome in chronic kidney disease: a consensus statement from the International Society of Renal Nutrition and Metabolism (ISRNM). J Ren Nutr. marzo de 2013;23(2):77-90.

24. Ikizler TA, Cano NJ, Franch $H$, Fouque $D$, Himmelfarb J, Kalantar-zadeh K, et al. Prevention and treatment of protein energy wasting in chronic kidney disease patients: a consensus statement by the International Society of Renal Nutrition and Metabolism. Kidney Int 2013 12;84(6):1096-107. doi:10.1038/ki.2013.147.

25. Rattanasompattikul M, Molnar MZ, Zaritsky JJ, Hatamizadeh $\mathrm{P}$, Jing J, Norris KC, Kovesdy CP, Kalantar-Zadeh K. Association of malnutritioninflammation complex and responsiveness to erythropoiesis-stimulating agents in long-term hemodialysis patients. Nephrol Dial Transplant 2013 07;28(7):1936-45 doi: 10.1093/ndt/gfs368.

26. Quero A, Fernández R, Fernández R, Gómez FJ. Estudio de la albúmina sérica y del índice de masa corporalcomomarcadoresnutricionalesen pacientes en hemodiálisis. Nutr. Hosp. 2015;31(3):1317-22 doi:10.3305/nh.2015.31.3.8084.
27. Demirci MS, Demirci C, Ozdogan 0, Kircelli F, Akcicek $F$, Basci $A$, et al. Relations between malnutrition-inflammation-atherosclerosis and volume status. The usefulness of bioimpedance analysis in peritoneal dialysis patients. Nephrol Dial Transplant. mayo de 2011;26(5):1708-16. doi: $10.1093 / \mathrm{ndt} / \mathrm{gfq} 588$.

28. Kovesdy $C P$, Kalantar-Zadeh K. Accuracy and limitations of the diagnosis of malnutrition in dialysis patients. Semin Dial. julio de 2012;25(4):423-7. doi:10.1111/j.1525-139X.2012.01097.x.

29. Toledo FR, Antunes AA, Vannini FC, D., Silveira $L V, A$., et al. Validity of malnutrition scores for predicting mortality in chronic hemodialysis patients. Int Urol Nephrol 2013;45(6):1747-52. doi 10.1007/s11255-013-0482-3.

30. Anand $S$, Chertow $G M$, Johansen $K L$, Grimes B, Kurella Tamura M, Dalrymple LS, et al. Association of self-reported physical activity with laboratory markers of nutrition and inflammation: the Comprehensive Dialysis Study. J Ren Nutr. noviembre de 2011;21(6):429-37 doi:10.1053/j. jrn.2010.09.007.

31. Sezer S, Bal Z, Tutal E, Uyar ME, Acar N0. Longterm oral nutrition supplementation improves outcomes in malnourished patients with chronic kidney disease on hemodialysis. JPEN J Parenter Enteral Nutr. noviembre de 2014;38(8):960-5.

32. Chen J, Peng H, Zhang K, Xiao L, Yuan Z, Chen $\mathrm{J}$, et al. The insufficiency intake of dietary micronutrients associated with malnutritioninflammation score in hemodialysis population. PLoS ONE. 2013;8(6):e66841. doi:10.1371/ journal.pone.0066841.

33. Beberashvili I, Azar A, Sinuani I, Kadoshi $H$, Shapiro G, Feldman L, Averbukh Z, Weissgarten J. Comparison analysis of nutritional scores for serial monitoring of nutritional status in hemodialysis patients. Clin J Am Soc Neprhol 2013 03;8(3):44351 doi:10.2215/CJN.04980512.

34. Streja E, Kovesdy CP, Molnar MZ, Norris KC, Greenland S, Nissenson AR, et al. Role of nutritional status and inflammation in higher survival of African American and Hispanic hemodialysis patients. Am J Kidney Dis. junio de 2011;57(6):883-93. doi:10.1053/j.ajkd.2010.10.050. 